\title{
UNA MIRADA DESDE LOS ESTUDIANTES DE PEDAGOGÍA AL PROCESODEFORMACIÓNPRÁCTICA: EL CASO DEL MODELO DEL AÑO 2000 DE LA UNIVERSIDAD CATÓLICA SILVA HENRÍQUEZ
}

\author{
Hernán Cofré, Claudia Vergara , María Inés Noguera , Alejandra \\ Silva
}

\begin{abstract}
RESUMEN
El presente trabajo resume los hallazgos más importantes de la investigación realizada sobre la opinión de los alumnos acerca del modelo de formación práctica en carreras de pedagogía de la Universidad Católica Silva Henríquez. El estudio, realizado entre los meses de septiembre de 2007 y julio 2008, se propuso identificar las características de los estudiantes en práctica profesional de ocho carreras de formación pedagógica. Para el logro de este propósito, se utilizó un enfoque metodológico cuantitativo, lo que incluyó la aplicación de un cuestionario con preguntas en escala likert a estudiantes que estuvieran en su último año de estudios. Los resultados muestran que los alumnos en práctica critican el desempeño de sus supervisores y de la propia universidad..
\end{abstract}

Palabras Clave: Estudiante de pedagogía, práctica profesional, programa de formación, escuelas

\section{A VIEW FROM PEDAGOGY STUDENTS REGARDING THE PROCESS OF PRACTICUM FORMATION: THE CASE OF THE 2000 MODEL OF UNIVERSIDAD CATÓLICA SILVA HENRÍQUEZ}

\section{ABSTRACT}

This paper summarizes the most important findings of the research on students' opinion about the model of practicum training for the programs at the Universidad Católica Silva Henríquez. The study, held between September 2007 and July 2008, set out to identify the characteristics of students in the practicum, in eight out of the 12 teacher training programs. To achieve this purpose, the authors used a quantitative methodological approach, which included implementation of a Likert scale questionnaire to students who were in their senior year. The results showed that students in practicum criticize the performance of supervisors and the university itself.

Keywords: Students, Practicum Formation, Teaching programs, Schools

* Doctor en Ciencias Biológicas. Académico de la Escuela de Educación Inicial, Universidad Católica Silva Henríquez, General Jofré 462. hcofre@ucsh.cl

Doctora en Ciencias de la Educación. Académica de la Escuela de Educación Inicial, Universidad Católica Silva Henríquez, General Jofré462.cvergara@ucsh.cl

Magíster (Ed) María Inés Noguera, Académica de la Escuela de Educación Inicial, Universidad Católica Silva Henríquez, General Jofré 462.mnoguera@ucsh.cl

Doctora (c) en Ciencias de la Educación. Académica de la Escuela de Educación Inicial, Universidad Católica Silva Henríquez, General Jofré 462. asilva@ucsh.cl 
Una mirada desde los estudiantes de pedagogía al proceso de formación práctica: el caso del modelo del año 2000 de la Universidad Católica Silva Henríquez - Hernán Cofré, Claudia Vergara, María Inés Noguera y Alejandra Silva

\section{Introducción}

Durante las últimas décadas, la educación a nivel nacional e internacional ha estado marcada por cambios sustantivos. Se observan cambios relacionados con la organización escolar, la toma de decisiones, el currículo, evaluaciones estandarizadas, estándares curriculares y el surgimiento de nuevas concepciones sobre el enseñar y aprender. En este contexto, la demanda a los centros educativos y, particularmente, a los docentes se está tornando cada vez más compleja. Los profesores deben ser capaces de preparar a los estudiantes para una sociedad y una economía que demanda autonomía en el aprendizaje y aptitud y motivación para seguir aprendiendo a lo largo de toda su vida (OECD 2004). La urgencia por buscar respuestas en la formación inicial se ha intensificado, entendiendo que el tipo de formación impartida debe estar mejor articulada con las demandas establecidas en la Reforma Educacional e identificarse con la inequidad de los resultados alcanzados (Informe de la OECD, 2004; Informe Comisión sobre Formación Inicial Docente, MINEDUC, 2005). Se reconoce la necesidad de re-pensar tanto la estructura actual de la formación inicial de los futuros docentes, como su formación práctica y la necesidad de construir una nueva identidad profesional (Korthagen et al., 2006). Es así como, en nuestro país, las universidades formadoras de profesores han implantado las prácticas progresivas en el entendido de que la práctica es el eje articulador entre la formación pedagógica y la disciplinaria, a la vez que facilitadora del proceso de construcción del saber docente.

En relación con la formación práctica, las investigaciones en América Latina muestran que los docentes reconocen que las prácticas han sido un componente importante en su formación, que les permite establecer relaciones con los centros educativos. Sin embargo, en la gran mayoría de los países, la proporción de tiempo asignada a la práctica en el aula es mínima en relación con el tiempo que se otorga a otras ramas de la formación como la pedagogía general y la formación disciplinar, salvo excepciones como Cuba, Uruguay y últimamente Chile (Ávalos, 2002). En Chile, en particular, existen pocos estudios 
sobre las prácticas que realizan los profesores en formación. Uno de los pocos datos sobre la percepción de los estudiantes de esta etapa de su formación, emerge del estudio realizado por Ávalos y Aylwin (2007). En esta investigación realizada a profesores neófitos o principiantes, con menos de 5 años de experiencia, se identificaron dos aspectos de su formación práctica que podrían estar influyendo en las debilidades que ellos reconocen tener en sus propias prácticas docentes. Estos aspectos eran, en primer lugar, que sus prácticas profesionales fueron muy poco observadas por sus profesores supervisores de la universidad y colaboradores de las escuelas, y en segundo lugar, ellos manifestaban que la experiencia práctica llegó muy al final de su formación como docente.

En este escenario, este estudio tuvo como objetivo general investigar las percepciones y necesidades de los estudiantes en el proceso de práctica profesional que se lleva a cabo en el último año de formación en la UCSH. Específicamente, nos interesó investigar las percepciones de los estudiantes sobre el desempeño del profesor colaborador, el desempeño del profesor supervisor, la utilidad de las prácticas tempranas y la calidad de su formación en general.

\section{Marco Teórico}

Características generales de los modelos de prácticas en el mundo

Existe una gran diversidad de fórmulas para preparar, en su aspecto práctico, a los futuros docentes, ya sea en términos de la duración, la cronología o el tipo de prácticas (Cofré et al., en revisión). Por ejemplo, en Finlandia, una de las naciones con mejores resultados en las evaluaciones internacionales como PISA y TIMMS posee solamente dos instancias de práctica profesional: una al finalizar su formación de licenciado en el tercer año de carrera, con cinco semanas de permanencia, y otra al finalizar su Master en educación en el último semestre del quinto año con una duración de 8 semanas (Jyrhämä, 2007; Niemi \& Jakku-Sihvonen, 2007). Esto contrasta con 
Una mirada desde los estudiantes de pedagogía al proceso de formación práctica: el caso del modelo del año 2000 de la Universidad Católica Silva Henríquez - Hernán Cofré, Claudia Vergara, María Inés Noguera y Alejandra Silva

otras naciones, dentro de las cuales existen ejemplos de universidades donde se trabaja con modelos con mucha mayor proporción del currículo cubierto por las prácticas (Murillo, 2006). Por ejemplo, los estudiantes de la Universidad Profesional STOAS de Holanda, un país que también tiene altos rendimientos en PISA y TIMMS, dedican el 60\% de su tiempo a las prácticas (Gómez, 2006). En general, en la mayoría de los países o Universidades que forman profesores, incluyen tiempos dedicados a prácticas cercanos a las 20 semanas durante toda la carrera (Inglaterra, Portugal, Canadá, Nueva Zelanda, Singapur, entre otros). De esta carga, también se puede decir, que cerca de I5 semanas son prácticas de tipo responsable o donde el estudiante se hace cargo completamente de la planificación y la enseñanza en el aula, mientras que el resto corresponden a prácticas de observación, donde muchas veces los estudiantes realizan investigaciones y entregan informes sobre la reflexión de los temas estudiados. Finalmente, también se observa que, en la mayoría de los modelos, existe un acercamiento progresivo del estudiante a las prácticas, donde la formación práctica y teórica se va entrelazando a través de los años (Cofré et al., en revisión).

\section{La visión y el rol de los estudiantes en su formación práctica}

Existe una amplia evidencia de que los profesores en formación, no siempre están conformes con la manera en que las instituciones de educación superior los preparan en el ámbito de la práctica (e. g. Korthagen, 200I; Smith \& Lev-Ari, 2005; Latorre, 2007). Algunas investigaciones realizadas en España muestran que los estudiantes critican la poca coordinación entre la Universidad formadora y la escuela que los recibe y entre los profesores colaboradores y los supervisores de práctica (Latorre, 2007). Sin embargo, también existen evidencias del impacto positivo que tienen las experiencias prácticas en los estudiantes para profesores (Smith \& Snoek, I996; Cameron \& Baker, 2004; Hascher et al., 2004; Smith \& Lev-Ari, 2005). Por ejemplo, Smith y Snoek (1996) reportaron que profesores en formación de Holanda e Israel declaran que las prácticas fueron la 
parte más valiosa de su formación como profesores. Por otra parte, existe conciencia, por parte de los estudiantes en práctica, de que el rol del profesor colaborador es de suma importancia (Atay, 2007). Asimismo, se ha demostrado que los alumnos en práctica también reconocen, en el supervisor de la Universidad, un importante apoyo para su desarrollo profesional (Smith \& Lev-Ari, 2005; Fayne, 2007; White, 2007). Específicamente, algunos estudios han detectado que los estudiantes valoran mucho los consejos o la retroalimentación específica que realiza el supervisor luego de las observaciones, sobre temas particulares de la práctica del estudiante (White, 2007). De hecho, recientemente Fayne (2007) ha mostrado que los alumnos en práctica reconocen que el profesor supervisor de la universidad y el profesor colaborador de la escuela les entregan diferentes aportes para su práctica. El profesor supervisor es valorado por dar apoyo emocional y académico, así como por ser un facilitador que permite el funcionamiento de la "tríada", mientras que el colaborador se valora por su experiencia y se ve como un modelo a seguir.

\section{Metodología}

Se utilizó un enfoque de tipo socioconstructivo con énfasis en el uso de instrumentos de tipo cuantitativo, orientados a la búsqueda de las características más importantes de la muestra estudiada y determinar las distintas variables que facilitan o dificultan la acción formadora de los docentes que acompañan a los estudiantes en práctica. La aproximación de carácter cuantitativo se expresa en la aplicación del cuestionario destinado a determinar el rol y funciones de los estudiantes en práctica o profesores en formación (Bryman 2004).

\section{Cuestionario y Variables estudiadas}

El cuestionario de los estudiantes en práctica constó de dos partes: una primera para recoger antecedentes descriptivos de carácter sociodemográfico de los estudiantes y, en general, sobre su experiencia práctica y luego una segunda parte con 48 ítemes en escala de apreciación. En esta segunda parte del cuestionario, se distinguieron 
Una mirada desde los estudiantes de pedagogía al proceso de formación práctica: el caso del modelo del año 2000 de la Universidad Católica Silva Henríquez - Hernán Cofré, Claudia Vergara, María Inés Noguera y Alejandra Silva

ámbitos: apreciación sobre el profesor supervisor, apreciación sobre los talleres de práctica, apreciación sobre el profesor colaborador y apreciación sobre la práctica en general. Cada uno de estos ámbitos formó parte de la variable dependiente. El valor de cada ámbito antes señalado, fue definido como la suma de todas las preguntas que lo conformaron. Para obtener esta suma, se le asignó un valor a cada categoría dentro de la escala de apreciación de cada pregunta. Los valores asignados a cada categoría fueron: Totalmente en desacuerdo= I, Parcialmente en desacuerdo $=2$, Parcialmente de acuerdo $=3 \mathrm{y}$ Totalmente de acuerdo $=4$. El resultado de estas sumas se relacionó con la variable independiente, nivel educativo al cual pertenecía el estudiante en práctica. Los Niveles Educativos fueron definidos como: $\mathrm{I}=$ Educación Diferencial, 2 = Educación Parvularia; 3 = Educación Básica, y 4 = Educación Media.

Fueron entregados 489 cuestionarios a estudiantes en práctica profesional durante el segundo semestre del año 2007, teniendo un retorno de 243 encuestas. Esto representó el 50\% de la muestra inicial. El retorno de cuestionarios en cada una de las carreras incluidas en el estudio fue: 9I de I45 en Pedagogía Básica (63\%), 24 de 50 en Educación Parvularia (48\%), 42 de 42 en Educación Diferencial (100\%), 39 de 80 en Pedagogía en Historia (49\%), I9 de 120 en Pedagogía en Educación Física (I6\%), 6 de 30 en Pedagogía en Educación Matemática y Computación (20\%); I I de I I en Pedagogía en Castellano (100\%) y I I de I I en Pedagogía en Inglés (100\%).

El cuestionario fue sometido a una validación por expertos en el área, los cuales sugirieron modificaciones que fueron acogidas en su totalidad.

Para el análisis de los cuestionarios, se utilizó el programa SPSS I5.0, el cual permite realizar diferentes agrupaciones de los datos. De esta manera, se pudo obtener la distribución de frecuencia y porcentajes para los aspectos descriptivos. 
Al realizar el análisis de confiabilidad para el cuestionario de alumnos en práctica, se obtuvo un $\alpha=0,9 \mathrm{I}$.

\section{Resultados}

\section{Aspectos demográficos y descriptivos}

De acuerdo a los datos sociodemográficos recuperados en los cuestionarios, se puede sostener que, en general, los alumnos en práctica son jóvenes cuya edad fluctúa entre los 22 y los 26 años, que en su mayoría son mujeres, que ingresaron los años 2003 ó 2004 a estudiar pedagogía, y que la mayoría de ellos proviene de colegios particulares subvencionados y, en menor medida, municipales. En cuanto a la apreciación que los estudiantes tienen de las actividades que se realizan en su práctica profesional y a la experiencia práctica que ellos poseen luego de estudiar 4 ó 5 años en la UCSH se puede desprender lo siguiente:

I.- La mayoría de los alumnos realizó actividades prácticas en escuelas y liceos municipales o en colegios particulares subvencionados (Fig. I). Muchos de ellos han tenido experiencia en sólo uno de estos tipos de establecimientos, mientras que alrededor de la mitad reconoce haber tenido alguna actividad práctica en dos tipos de establecimientos (los que en su mayoría son municipales y particulares subvencionados). Es decir, los alumnos de la UCSH, tienen un escaso contacto con colegios particulares pagados a lo largo de su experiencia práctica dentro de la universidad.

2.- En la misma línea de análisis, cuando se les preguntó en cuántos establecimientos han tenido alguna actividad práctica dentro de su carrera, la mayoría contestó que recuerda haber visitado entre 2 y 4 distintos establecimientos (Fig. 2). Es decir, los alumnos de la UCSH, en general, han visitado menos de 4 establecimientos educacionales distintos a través de su experiencia práctica. 
Una mirada desde los estudiantes de pedagogía al proceso de formación práctica: el caso del modelo del año 2000 de la Universidad Católica Silva Henríquez - Hernán Cofré, Claudia Vergara, María Inés Noguera y Alejandra Silva

Fig. I. Número de alumnos que han tenido contacto con los diferentes tipos de establecimientos que se pueden reconocer en el sistema educativo nacional.

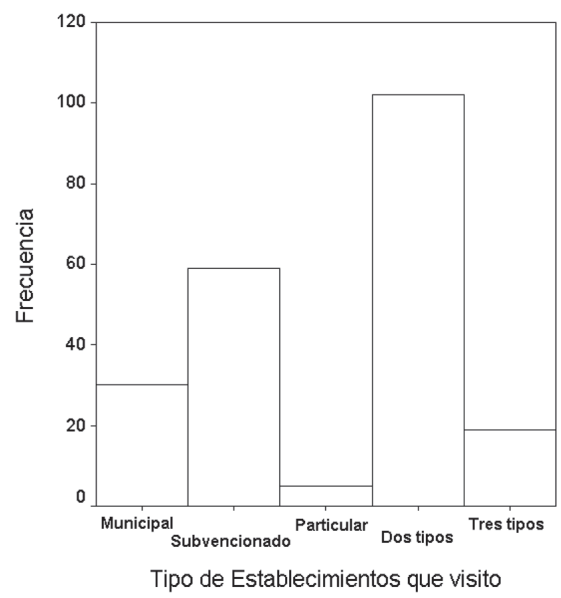

Fig. 2. Número de establecimientos distintos en los cuales los alumnos en práctica profesional recuerdan haber tenido alguna actividad relacionada con la práctica pedagógica.

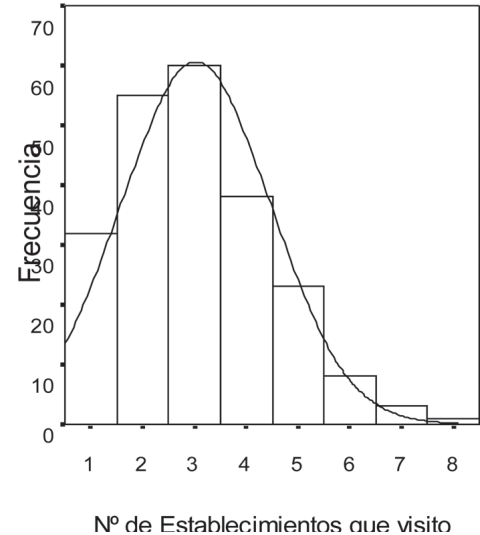

3.- En cuanto a las características de la práctica profesional, se observa que la mayoría de los profesores en formación, dice que más del 70\% del tiempo utilizado en su práctica profesional estuvieron a cargo del curso. No obstante, al analizar las respuestas de acuerdo a los distintos niveles educativos, se pueden apreciar diferentes patrones de respuesta (Fig. 3). Específicamente, los alumnos que estudian las carreras de Pedagogía en Educación Parvularia y Educación Diferencial son los que se hacen cargo de un curso en una mayor proporción del tiempo ( $>90 \%)$. 
Por otro lado, dentro del grupo de los alumnos en práctica que pertenecen a la carrera de Pedagogía Básica, existen al menos 30 que estuvieron a cargo del curso menos del 70\% del tiempo de práctica. Finalmente, gran parte de los profesores en formación de las carreras de Educación Media, responden que se hacen cargo de un curso sobre el 90\% del tiempo de su práctica.

Otro de los aspectos consultados fue el acompañamiento que los alumnos en práctica tienen por parte de sus profesores colaboradores y supervisores. En cuanto a los profesores colaboradores, cerca de la mitad de los alumnos encuestados dijo que éstos observaron más del $90 \%$ de las clases que ellos realizaron (Fig. 4). No obstante, estos resultados muestran que existe una proporción importante de alumnos que son observados menos del $70 \%$ de las horas en que están encargados de curso (aproximadamente 40 alumnos). Este patrón difiere entre niveles educativos, si por ejemplo, existe un número alto de alumnos en práctica que corresponde a pedagogías en Educación Media, que declaran haber sido observados menos de un 50\% de las horas de práctica. Este patrón también se observa en el grupo de alumnos de Educación Diferencial. En contraste, la mayoría de los alumnos de Pedagogía Básica declara que el profesor colaborador del establecimiento permanece más del $90 \%$ del tiempo observando su desempeño a cargo de curso.

Fig. 3. Proporción de la práctica profesional en que los profesores en formación se hacen cargo de curso. Los números corresponden a: $\mathrm{I}=<50 \% ; 2=50-69 \% ; 3=70-89 \% ; 4=90$ 99\%; $5=100 \%$. La Figura muestra como ejemplos las respuestas de estudiantes de Educación Media y Educación Básica.

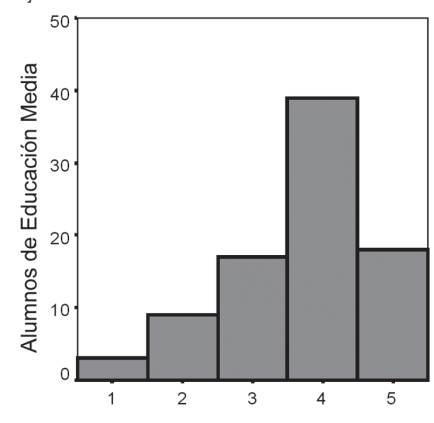

$\%$ de la Práctica a cargo del curso

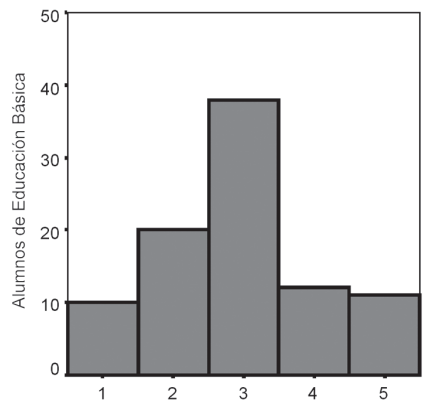

$\%$ de la Práctica a cargo del curso 
Una mirada desde los estudiantes de pedagogía al proceso de formación práctica: el caso del modelo del año 2000 de la Universidad Católica Silva Henríquez - Hernán Cofré, Claudia Vergara, María Inés Noguera y Alejandra Silva

En cuanto a los profesores supervisores, la mayoría de los alumnos en práctica señala que éstos los visitan entre 2 y 3 veces en el semestre. No obstante, al analizar sus respuestas por nivel educativo, se observa que existen notables diferencias a este respecto (Fig. 5). Por ejemplo, una parte importante de los alumnos de Educación Media señala que sus profesores supervisores los visitaron sólo en una ocasión. En el otro extremo del espectro, la mayoría de los alumnos de educación diferencial señala que sus supervisores los visitan 4 o más veces en el semestre.

Fig. 4. Proporción de la práctica profesional en que los profesores colaboradores observaron a los alumnos en práctica. Los números corresponden a: $\mathrm{I}=<50 \% ; 2=50-69 \% ; 3=70-89 \%$; $4=90-99 \% ; 5=100 \%$. La Figura muestra como ejemplos las respuestas de estudiantes de Educación Básica y Educación Diferencial.
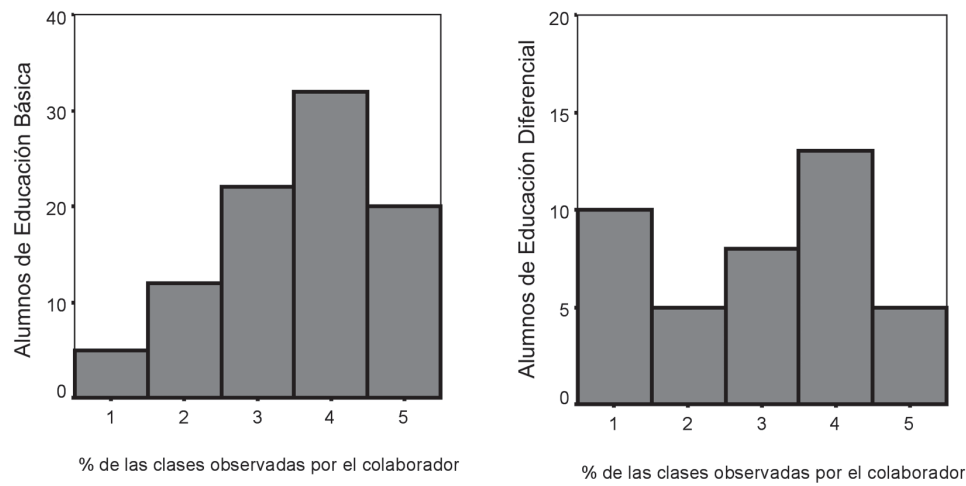

Fig. 5. Número de visitas que los profesores supervisores destinaron a observar a los alumnos en práctica. Los números corresponden a: $0=$ ninguna visita $I=$ una visita; $2=2$ a 3 visitas; $3=>$ de 3 visitas.

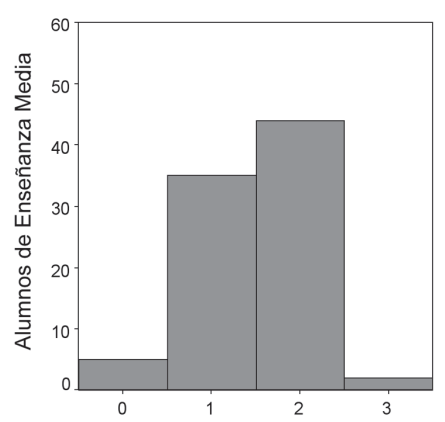

Número de visitas del supervisor

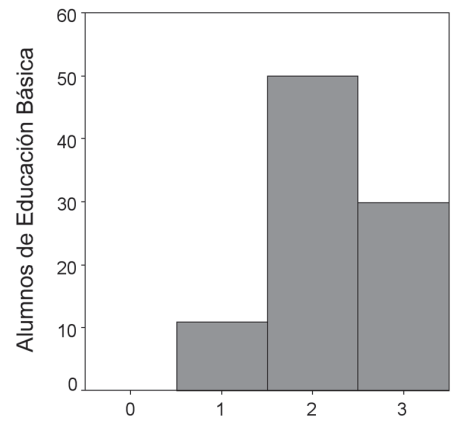

Número de visitas del supervisor 


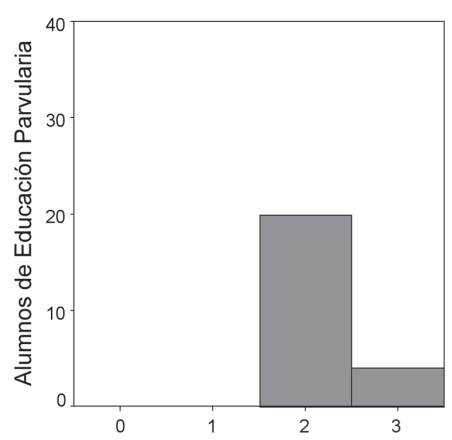

Número de visitas del supervisor

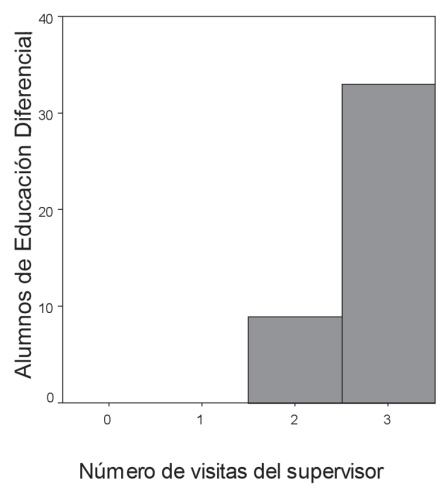

Número de visitas del supervisor

Percepción del profesor en formación sobre las actividades prácticas realizadas en la $\mathrm{UCSH}$

\section{Desempeño del profesor Supervisor}

En cuanto al desempeño del profesor supervisor, en general, los alumnos en práctica tienen una buena percepción de su gestión. Sin embargo, existe un número pequeño de alumnos que no está de acuerdo con muchas de las afirmaciones de un buen desempeño. Esta discrepancia de percepciones no es al azar, ya que existen diferencias significativas entre las percepciones de los alumnos de las diferentes carreras de los diferentes niveles educacionales (Fig. 6). Los alumnos de carreras de Enseñanza Media tienen percepciones más negativas que alumnos de Educación Básica, y estos alumnos, percepciones más bajas que alumnos de Educación Parvularia o Educación Diferencial. 
Una mirada desde los estudiantes de pedagogía al proceso de formación práctica: el caso del modelo del año 2000 de la Universidad Católica Silva Henríquez - Hernán Cofré, Claudia Vergara, María Inés Noguera y Alejandra Silva

Figura 6. Comparación del grado de acuerdo o desacuerdo del desempeño del profesor supervisor de práctica entre niveles educativos $(F=6.38 ; \mathrm{p}<0.00 \mathrm{I})$. Niveles educativos: I= Educación Diferencial, 2 = Educación Parvularia; 3 = Educación Básica, y 4 = Educación Media.

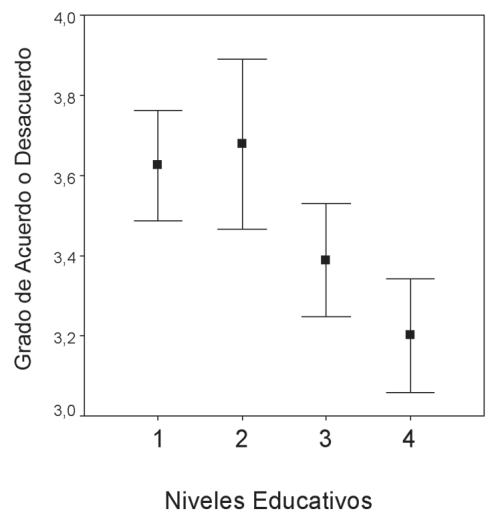

\section{Función de los Talleres de Práctica}

En cuanto a la función que cumplen los Talleres de Prácticas, en general, los alumnos en práctica están de acuerdo en que éstos son una instancia para recibir apoyo en cuanto a la formación pedagógica. Sin embargo, existe cerca de un $15 \%$ de los alumnos que no está de acuerdo con muchas de las afirmaciones que describen el Taller de Práctica como una actividad donde se recibe formación. Esta discrepancia de percepciones no es al azar, ya que existen diferencias significativas entre las percepciones de los alumnos de las diferentes carreras de los diferentes niveles educativos (Fig. 7). Los alumnos de carreras de Enseñanza Media y los de Pedagogía Básica, están menos de acuerdo sobre el tema que alumnos de Educación Parvularia o Educación Diferencial. Por lo tanto, se puede pensar que el Taller de Práctica en estas dos últimas carreras, cumple principalmente una función de formación. 
Figura 7. Comparación del grado de acuerdo o desacuerdo con la apreciación de los talleres de práctica como una instancia formativa, por Niveles educativos ( $F=3,1 ; p<0.028$ ).

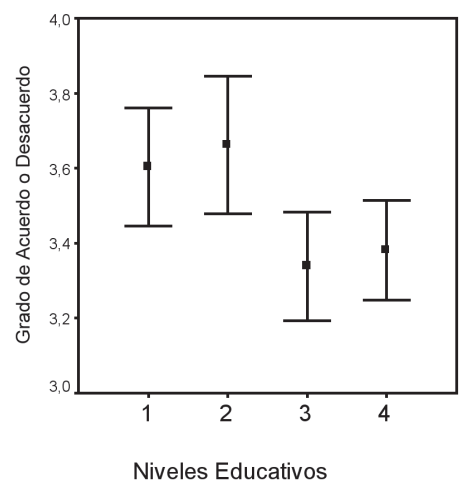

\section{Desempeño del profesor colaborador}

En cuanto al desempeño del profesor colaborador, la mayoría de los alumnos en práctica tiene una buena opinión, tanto en aspectos formales, como en aspectos formativos. Sin embargo, existen algunos alumnos que no están de acuerdo con las afirmaciones de un buen cometido por parte del profesor colaborador. Estas opiniones no parecen venir de alumnos de ninguna carrera en particular, ya que no existe diferencia significativa entre las respuestas de alumnos en práctica de las diferentes carreras. Por otro lado, cuando se preguntó a los alumnos si existían conductas poco adecuadas por parte de sus profesores colaboradores, existe una parte importante de los estudiantes que está de acuerdo con estas aseveraciones (Fig. 8).

Figura 8. Grado de acuerdo o desacuerdo con un mal desempeño de profesor colaborador.

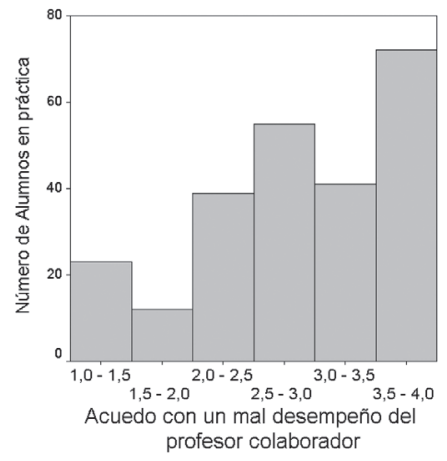


Una mirada desde los estudiantes de pedagogía al proceso de formación práctica: el caso del modelo del año 2000 de la Universidad Católica Silva Henríquez - Hernán Cofré, Claudia Vergara, María Inés Noguera y Alejandra Silva

\section{Discusión y Conclusiones}

La principal reflexión que emerge luego de analizar los datos y la información recopilada en esta investigación, a la luz de la teoría existente, es que el proceso de prácticas profesionales que realizaban los alumnos de la UCSH hasta el año 2007 coincide con los procesos que se daban en el pasado en otras partes del mundo, y que hoy en día están siendo reemplazados paulatinamente por procesos más significativos. Por ejemplo, los alumnos en práctica de la UCSH critican el desempeño de los supervisores y de la propia universidad, ya que se sienten solos en el centro de práctica, al cual muchas veces llegan por sus propios medios. Esto se ha observado en otras partes del mundo como España, Israel o Turquía, donde también se critica la poca coordinación entre la Universidad y los centros de práctica. Esta crítica es muy válida ya que existen evidencias sólidas de que una buena coordinación entre la Universidad y los centros receptores es una de las claves para tener prácticas de calidad, por lo que los centros de formación de profesores están poniendo especial énfasis en mejorar este tema (Murillo 2006).

Otra evidencia directa del esquema tradicional de prácticas que se daba en la UCSH, es la percepción de los estudiantes de que la formación práctica que les da la Universidad sólo llega al final de su carrera, visión que también comparten en alguna medida supervisores y colaboradores, quienes declaran que los alumnos llegan con poca experiencia de aula a sus prácticas. En este sentido, parece no haber sido efectivo el modelo de prácticas tempranas y progresivas adoptado por la UCSH a comienzos de la década, en consonancia con los cambios ocurridos en los principales centros de formación alrededor del mundo (e.g. Murillo 2006). Hemos señalado, que la concepción tradicional de dependencia de la práctica entendida como la aplicación de la teoría llevaba a que las prácticas se desarrollaran al finalizar la formación. La reformulación de esta idea en los últimos años hace que los centros de formación de profesores, busquen combinar la teoría y la práctica desde los primeros momentos de la formación y a lo largo de toda ella. Algunos ejemplos notables de modelos innovativos de formación que han adoptado esta modalidad 
son la Universidad profesional STOAS en Holanda (Gómez, 2006), el Proyecto Veredas de Brasil (Jamil, 2006) y el cambio llevado a cabo en la Universidad Humboldt (Schimpf-Herken, 2006). Por lo tanto, la UCSH está llamada a buscar cómo implementar en forma exitosa su modelo de prácticas tempranas y progresivas para la formación de profesores, más que a abandonar esta iniciativa.

Aunque existen pocos antecedentes, es probable que este tipo de práctica tradicional aún se esté dando en el resto de las universidades del sistema chileno (Montecinos, et al., 2002; Ávalos y Aylwin, 2007; Silva-Peña et al., 2008; véase además, Cornejo y Fuentealba, 2008), pese a los cambios en la formación práctica que fueron impulsados por el Programa de Fortalecimiento de la Formación Inicial Docente (PFFID) a fines de los '90. Por ejemplo, Ávalos y Aylwin (2007) mostraron que profesores principiantes, con menos de 5 años de experiencia, manifestaron que sus prácticas profesionales fueron poco observadas por los profesores supervisores de la universidad y por los profesores que los recibían en las escuelas y que, por tanto, la retroalimentación y el apoyo por parte de ellos fue escasa. Por otro lado, existen algunas evidencias de que muchos profesores supervisores de universidad no cumplen una labor de acompañamiento y desarrollo del estudiante en práctica, sino que más bien, están en una función evaluativa e, incluso a veces, una función de contención emocional o de apoyo disciplinar de los estudiantes (Montecinos, 2002; Noguera et al., en preparación). Un primer paso en el mejoramiento del desempeño de estos supervisores es lo que ha realizado la UCSH, donde expertos en el tema de la supervisión han comenzado a realizar capacitaciones en algunas de las carreras de pedagogía (Correa, 2007). Finalmente, con relación a los pocos datos sobre el papel del profesor colaborador en la formación de profesores en Chile en otras universidades, es probable que muchos de ellos adopten una posición de hospedero, en términos de que no se comprometen en la formación del futuro docente, y sólo se limitan a "prestar" el curso a los estudiantes en práctica (Ávalos y Aylwin, 2007; SilvaPeña et al., 2008). Este tipo de comportamiento es absolutamente comprensible, ya que, en estos momentos, no existe una retribución 
Una mirada desde los estudiantes de pedagogía al proceso de formación práctica: el caso del modelo del año 2000 de la Universidad Católica Silva Henríquez - Hernán Cofré, Claudia Vergara, María Inés Noguera y Alejandra Silva

gubernamental a aquellos profesores que colaboran en la formación de los estudiantes de pedagogía, algo que sí ocurre en algunos países desarrollados (Correa, 2007), por lo que es responsabilidad única de las universidades el generar convenios y relaciones estrechas con las escuelas para retribuir a ellas y a sus profesores el aporte a la educación de los profesores en formación.

A la luz de nuestros resultados, y basándonos en las experiencias exitosas de países con sistemas educativos reconocidamente eficientes, se pueden proponer las siguientes medidas de mejoramiento de la formación práctica de los estudiantes de pedagogía de la UCSH:

$I^{\circ}$ La UCSH debería continuar con las investigaciones sobre el proceso de prácticas profesionales, ya que es la principal forma de monitorear e ir implementando cambios que tiendan a mejorar cada vez más este proceso (Zeichner, I995, 2005). La formación práctica de profesores, es una de las principales líneas de investigación en educación hoy en día en el mundo (e.g. Atay, 2007; Fayne, 2007; Braund en preparación), y mejorarla repercutirá directamente en el desempeño de los profesores recién egresados y, finalmente, en el aprendizaje de sus alumnos (Barber \& Mourshed, 2007).

$2^{\circ}$ La UCSH debería reforzar los convenios y el trabajo conjunto con escuelas, colegios y liceos, pues son una parte fundamental dentro de la formación de los estudiantes en práctica (Zeichner, 2005; Braund en preparación). Como se ha demostrado en Inglaterra y otros países desarrollados, la mejor forma de estructurar una experiencia práctica es a través de darle la misma importancia a los aprendizajes que logra el estudiante en práctica en la escuela, como los que logra en la universidad. Una de las formas más efectivas de lograr que las escuelas y profesores guía o colaboradores se involucren en el logro de estos aprendizajes es mostrarles los beneficios que les puede reportar la interacción con el profesor en formación (Braund, 200I; Braund en preparación).

$3^{\circ} \mathrm{La}$ UCSH debería reforzar el trabajo con los profesores supervisores de la universidad y con los profesores guías de las 
escuelas, toda vez que ellos se involucren más intensamente en la preparación del profesor en formación. Se sabe que estos actores cumplen roles diferentes e irremplazables dentro de la formación práctica (Cofré et al., en revisión), por lo cual se debería intensificar el trabajo de capacitación de ambos, en los próximos períodos de práctica profesional.

$4^{\circ}$ Finalmente, la UCSH debería organizar al menos una experiencia de formación práctica temprana (alrededor del segundo año) en la cual los alumnos y alumnas conozcan en profundidad el quehacer pedagógico en los centros educativos (Cofré, 2009). Estas prácticas tempranas son comunes en países con buenos resultados internacionales (e.g. Canadá, Inglaterra, Finlandia, entre otros), las cuales son mucho más que simples observaciones, sino que son procesos de reflexión y/o investigación guiados por un profesor supervisor de universidad y un profesor guía en la escuela (Cofré et al., en revisión).

Estas sugerencias, junto a los esfuerzos del nuevo proyecto Mecesup de mejoramiento de las prácticas al interior de la universidad, deberían mejorar sustancialmente la formación práctica impartida por la UCSH, en el corto y mediano plazo. El resto de las universidades chilenas debería mirar en esta misma dirección, si queremos mejorar la formación de nuestros futuros profesores. No obstante, es claro que, para mejorar las prácticas de los profesores novatos que egresarán de las universidades chilenas en los próximos años, es igualmente urgente mejorar las condiciones laborales en las que ellos se insertarán. Principalmente, se deberían aumentar los salarios iniciales y disminuir las horas frente a curso, para así aumentar las horas efectivas de reflexión y preparación de clases. Estas dos medidas, son características casi universales en la mayoría de los países con los mejores desempeños en evaluaciones internacionales como PISA y TIMMS (Barber \& Mourshed, 2007). 
Una mirada desde los estudiantes de pedagogía al proceso de formación práctica: el caso del modelo del año 2000 de la Universidad Católica Silva Henríquez - Hernán Cofré, Claudia Vergara, María Inés Noguera y Alejandra Silva

\section{Bibliografía}

Atay, D. (2007). "Beginning teacher efficacy and the practicum in an EFL context". Teacher Development, II: 203-2I9.

Ávalos, B. (2002). Profesores para Chile: Historia de un Proyecto. Santiago: Ministerio de Educación.

Ávalos, B. y Aylwin P. (2007). "How young teachers experience their professional work in Chile". Teaching and Teacher Education, 23: p. 515-528.

Barber, M. y Mourshed M. (2007). "How the World's Best-Performing School Systems Come Out On Top. McKinsey \& Company, Social Sector Office”. Recuperado en: http://www.mckinsey.com/clientservice/ socialsector/resources/pdf/Worlds_School_Systems_Final.pdf.

Braund, M. (200I). "Helping primary student teachers understand pupils'learning: exploring the student-mentor interaction", Mentoring and Tutoring, 9: p. 189-200.

Bryman, A. (2004). Social research methods (2nd ed.). New York: Oxford University Press.

Cameron, M. y Baker R. (2004). "Research on Initial Teacher Education in New Zealand: 1993-2004". Literature Review and Annotated Bibliograpby. New Zeland Council for Educational Research, Wellington.

Cofré, H. (2009). Informe de Asistencia Técnica Internacional 2. Proyecto MECESUPUCSH. UCS0705.

Fayne, H. R. (2007). "Supervision from the Student Teacher's Perspective: An Institutional Case Study Studying” Teacher Education 3: p. 53-66.

Gómez, S. M. (2006). "STOAS Universidad Profesional y la innovación en la formación inicial de docentes". En Murillo F. J. (ed), Modelos Innovadores en la formación inicial docente. Oficina Regional de Educación para América Latina y el Caribe.

Hascher, T.; Cocard, Y. y Moser, P. (2004). "Forget about theory-practice is all? Student teachers' learning in practicum”. Teachers and Teaching, I0: p. $623-637$.

Jamil C. R. (2006). "La formación de profesores en Minas Gerais, Brasil". En: Murillo F. J. (ed), Modelos Innovadores en la formación inicial docente. UNESCOOficina Regional de Educación para América Latina y el Caribe.

Jyrhämä, R. (2007). "The function of practical Studies in teacher education". En: Jakku-Sihvonen and Niemi H. (eds), Researc-based teacher education in Finland: reflections by finnish teacher educators. Finish Educational Research Association. 
Korthagen, F. (200I). Linking Practice and Theory. Lawrence Erlbaum, USA.

Korthagen, F.; Loughran, J. y Russell, T. (2006). "Developing fundamental principles for teacher Education Programs and Practices”. Teaching and Teacher Education, 22, p. 1020-I04I.

Latorre, M. J. (2007). "El potencial formativo del prácticum: cambio en las creencias que sobre la enseñanza práctica poseen los futuros maestros". Ministerio de Educación España. Revista de Educación, p. 249-273.

Murillo, F. J. (2006). "Panorámica general de las aportaciones innovadoras". En Murillo F. J. (ed), Modelos Innovadores en la formación inicial docente. UNESCOOficina Regional de Educación para América Latina y el Caribe.

MINEDUC, (2005). Informe Comisión sobre Formación Inicial Docente. Serie Bicentenario.

Niemi H. y Jakku-Sihvonen, R. (2007). "Research Based teacher education". In Jakku-Sihvonen and H. Niemi (eds). Research based teacher education in Finland: reflections by finnish teacher educators. Finish Educational research Association.

OECD (2004). "Revisión de Políticas Nacionales de Educación", Chile.

Schimpf-Herken, I. (2006). "El modelo de formación de docentes inicial de la Universidad Humboldt de Berlín”. En Murillo F. J. (ed), Modelos Innovadores en la formación inicial docente. UNESCO-Oficina Regional de Educación para América Latina y el Caribe.

Smith, K. y Snoek, M. (I996). "Dutch and Israeli student teachers' views on their future roles as teachers", paper presented to the Annual Association of Teacher Education in Europe, Glasgow, Scotland.

Smith, K., y Lev-Ari L. (2005). "The place of the practicum in preservice teacher education: the voice of the students". Asia-Pacific Journal of Teacher Education, 33: p. 289-302.

White, S. (2007). "Investigating Effective Feedback Practices for Pre-service Teacher Education Students on Practicum”, Teaching Education, I8: p. 299-3II.

Zeichner, K. (I995). "Reflections of a Teacher Educator Working for Social Change", in Russell, $\mathrm{T}$ and Kotthagen,F, 2 Teachers who Teach Teachers: Reflections on teacher Education, The falmer Press, p. II-24.

Zeichner, K. (2005). "Becoming a teacher educator: a personal perspective". Teaching and Teacher Education, 2I: p. II7-I24. 